ISSN 1870-4069

\title{
Towards a Natural Language-based Dialog Model as an Assistant Tool for Dementia Therapy
}

\author{
María J. Somodevilla, Víctor Manuel Morales-de-Jesús \\ Benemérita Universidad Autónoma de Puebla \\ Facultad de Ciencias de la Computación, Puebla, Mexico \\ mariajsomodevilla@gmail.com, victor.morales@cs.buap.mx
}

\begin{abstract}
Reminiscence therapy is a treatment of dementia that mitigates the unstable psychological states of a person with dementia, through conversations between patient and caregiver that evoke past experiences focusing on the patient's long-term memory. It is recommended that a person with dementia receive this type of therapy constantly. In this work, we propose the design of a dialog model that generates personalized conversations between a conversational system and patients with Alzheimer's disease, with the aim to assist their caregivers in providing this type of therapy. The proposed model will be capable of getting a semantic interpretation about what the user is saying, identifying the type of dialog, also it will use information from the patient's life history and lifestyle to generate the conversations. During the dialog between the system and the patient, the model searches for relevant information related to the patient's history through the use of ontologies to enrich and develop a further conversation.
\end{abstract}

Keywords. Dialog model, dementia, conversational systems, reminiscence therapy.

\section{Introduction}

Dementia is a neurodegenerative and progressive condition characterized by alterations in cognitive processes, behavior, mood, and the ability to perform everyday activities [7]. Alzheimer's disease (AD) is the most common type of dementia in the world and is also one of the main reasons for disability in elderly people after the age of 65 , generating dependence on those who suffer this affection. By the year 2010, there were 35.5 million people with dementia worldwide and it is predicted that by 2030 this number will increase to 65.7 million and 115.4 million by 2050 [17]. Therefore, the social cost of dementia is already enormous.

Following this trend, accelerated growth in the population of older adults is expected within the next 20 years in Mexico. According to some estimations, it is expected that during the 2000-2030 period the population in Mexico aged 0 
to 14 will have a decrease of $14 \%$, population aged 15 to 64 , present a growth of $47 \%$, while population aged over 64 will present a disproportionate growth of $300 \%$ [4]. Thus, a significant number of Mexican people will be elderly people, for example, while in 1970 there were 5 to 7 older adults versus 100 young people, it is estimated that by 2050 there will be 139 adults aged over 65 versus 100 young people. The analysis of this data is important because it reveals a need for the transformation of the care requirements for this population group due to its accelerated rate of growth. A population from which almost one third will be elderly people with a diverse level of dependence [7], and as we mentioned above, the main cause of dependency in old age is precisely dementia.

This situation is disturbing because it has been estimated that Mexico is one of the Latin American countries with the highest prevalence of the AD with 7.3 $\%$ [11]. Which means that from the 13 million adults aged over 60 who currently live in our country, approximately 1.5 million suffer the AD and it is estimated that by 2050 if not before, the number of Mexicans affected by the AD will increase more than 3.5 million [7].

Although currently, dementia is not a curable disease, it is needed that people suffering from some type of dementia such as Alzheimer's disease can preserve a good quality of life. In this sense, there are both pharmacological and non-pharmacological treatments focused on the reduction of the psychological, behavioral and cognitive impairment symptoms that a person with AD presents. It is preferable that dementia treatments begin with a non-pharmacological intervention, because of this type of treatments promote the use of different methods and techniques to provide physical and emotional safety to the patient without the side effects of pharmacological treatments [8]. Through the implementation of appropriate environments, stimulating tasks and therapies applied according to the specific needs of each patient, the effect of this type of intervention can be maximized [5].

Reminiscence therapy is among this type of intervention, in which a conversation is established between the patient and their caregiver about activities and experiences from the past, where the patient is guided chronologically through their life experiences [19]. This type of therapy is based on the patient's long-term memory using information on their life history, that is, information that is familiar to the patient and relatively easy to remember, since during the mild and moderate phase of the AD the short-term memory is affected mainly. In different studies, the benefits of subjecting patients with dementia to this type of therapies have been studied and it was observed that carrying out constantly this type of conversations supports to enhance the unstable and emotional psychological states of the patients, increasing their sociability and self-confidence $[9,19,6]$.

However, for most people with the $\mathrm{AD}$, it is unreasonable to consult a professional doctor or therapist every day. Also, even for caregivers, the time and effort spent on the counseling are quite limited since they have many others supports to do [14]. In this sense, the objective of this work focuses on the creation of a dialog model capable of generating personalized conversations between a conversational system and patients with $\mathrm{AD}$, based on information 
about the patient's life history to support caregivers and therapists to provide reminiscence therapy constantly with the purpose of mitigating emotional and psychological symptoms such as anxiety and depression generated by the AD and help to preserve the quality of life of the patient.

\section{Related work}

A brief review of the literature is presented, as well as a comparison with those proposals considered similar to this work, highlighting the constraints and differences detected with the proposed approach.

It is remarkable to mention that a large number of research proposals focus on the diagnosis of dementia through the classification of symptoms and identification of distinctive features for early detection of dementia. Tanaka et al. [16] proposed a computer avatar with spoken dialog functionalities that produces spoken queries based on the mini-mental state examination (MMS), the Wechsler memory scale-revised, and other related neuropsychological questions. They recorded the interactive data of spoken dialogues and extract different audiovisual features, then two machine learning algorithms were used achieving a 0.93 detection performance rate. Chinaei et al. [2] analyzed several linguistics features that are verbal indicators of confusion in AD like vocabulary richness, parse tree structures, and acoustic cues. They applied several machine learning algorithms to identify dialog-relevant confusion from speech with up to $82 \%$ accuracy.

On the other hand, there are approaches that promote the use of assistive technology to help people with dementia with activities of daily living (ADLs) $[20,13,18,8]$ and support different kinds of therapy to address psychological, emotional and behavioral symptoms of dementia $[1,3,14,15]$.

Yasuda et al. [20] developed a remote reminiscence conversation and schedule prompter system via videophone to improve psychological stability and to assist individuals with dementia to perform household tasks. They observed that psychological stability of 1 patient persisted for 3 hours after remote conversations. Futhermore, motivational promter videos were added and they found that the task completion rate afforded by the revised schedule prompter system was $82 \%$. This type of proposals are compared against other instructions strategies in [10]. Ruszicz et al. [13] built a mobile robot, call ED, intented to assist with ADLs through visual monitoring and verbal prompts. They analyzed speech-based interactions between $\mathrm{ED}$ and each of 10 older adults with $\mathrm{AD}$ as the latter complete daily tasks in a simulated home environment. Their analysis reveals that across the verbal behaviors that indicate confusion, patients with $\mathrm{AD}$ are very likely to simply ignore the robot, which accounts for over $40 \%$ of all such behaviors.

Wolters et al. [18] analysed the interaction between people with dementia and a simulated intelligent cognitive assistant (ICA) that support people with dementia who need performing everyday tasks by detecting when problems occur and providing tailored and context-sensitive assistance. They conducted 
three focus groups with people with dementia, carers, and older people without a diagnosis of dementia. Analysis of the focus data showed that voice and interaction style should be chosen based on the preferences of the patients and it is crucial that the user can personalise the ICA themselves.

Similarly, Navarro et al. [8] implemented an assisted cognition system and analyzed the results on the use an adoption of the system to support occupational therapy and evaluated the adoption and effectiveness of the system to ameliorate challenging behaviors and reducing caregiver burden. They conducted an in situ evaluation with two caregiver-patinent dyads and it was observed that intervention personalization and touch-based interface encouraged the adoption of the system, helping reduce challenging behaviors in people with dementia and caregiver burden.

Casey et al. [1] performed a qualitative study wich describes how people with dementia and other key stakeholders could help to design a companion robot (MARIO). In their research they identified elements of importance to end users that make a companion robot acceptable to them and more likely that they would engage and interact with it. At the end, a relevant aspect they found is that people with dementia would like MARIO to have access to their life history, their interests, and hobbies and use this information to foster conversations and reminiscence about events that they could remember more easily. Cruz-Sandoval et al. [3] designed a semi-autonomous agent, called Eva. The agent is capable of participating in simple conversations with the coordination of a human operator to be perceived as an engaging speaker. Eva implements non-pharmacological interventions such as cognitive games and music therapy. They conducted a preliminary evaluation with a group of caregivers $(n=8)$ obtaining quantitative and qualitative results that were used to asses the social abilities of the robot.

As was above-mentioned, a person with dementia should receive these interventions as often as possible. In this sense, virtual agent technology is considered a good alternative to offer daily dementia counseling even at home. Sakakibara et al. [14] proposed a method that dynamically generates personalized dialogs for individual people with dementia. Using the patient's life history the system chooses an appropriate conversation. During the conversation, the system finds new information in linked open data (LOD) relevant to the response and uses it to develop futher conversation. However, their prototype system only implements the personalized dialog for the user's birthplace.

Since language comprehension and production is coparatively well-preserved in people with mild to moderate $\mathrm{AD}$, proposals using dialog systems or voice interfaces to support non pharmacological dementia treatments like cognitive assistance and therapy, have shown be helpful and well adopted. However, in most analyzed proposals, generic dialogs are used, that is, the same conversation is used for all patients, so they do not provide the benefits that have been observed in conversations where the dialog is personalized to each patient as in reminiscence therapy. In this paper, we propose an approach that aims generate personalized dialogs to support reminiscence therapy. 


\section{Proposed Approach}

In this section, we mention our proposal that will be carried out through this work. Firstly, the process of developing the dialog model is described, and then how this dialog model will be integrated in a conversational system is showed.

\subsection{Dialog model description}

The construction of the dialog model considers different stages according to the particular tasks that need to be performed in each stage. Figure 1 shows the process for the construction of the dialog model.

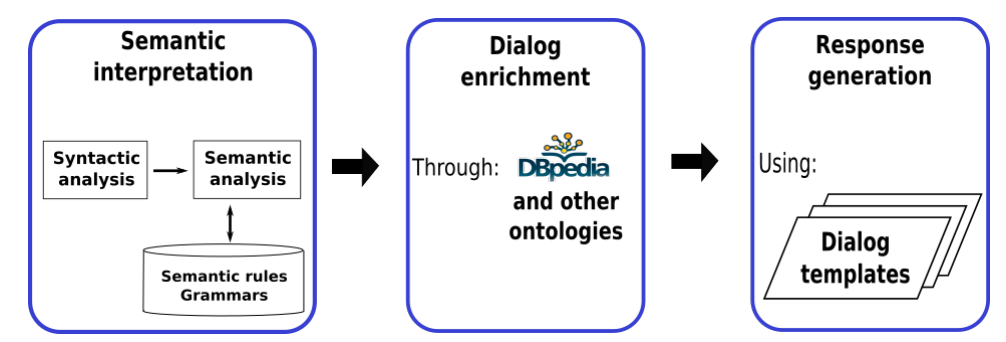

Fig. 1. Development of the dialog model.

Initially, it is necessary that the dialog model will be capable of getting a semantic interpretation about what the user is saying, this is, that the model will understand the type of dialog (question, a request of information, affirmation, etc.), as well as the meaning of what the user said. Therefore, the first thing that is proposed is a syntactic analysis of the sentences with the objective of grammatically labeling each word of the sentence. Subsequently, it is intended to perform a semantic analysis by defining a set of semantic rules according to the patterns detected within the grammar labels added in the syntactic analysis.

As result from the semantic analysis, it is expected to detect some "semantic attributes", this attributes then will be taken to retrieve relevant information according to the patient's life history through the use of diverse ontologies such as DBpedia $^{1}$. Finally, the generation of the response is carried out using appropriate dialog templates according to the information retrieved from the ontologies.

Because it is planning that the created model be able to generate a personalized dialog to each patient, it is necessary to have the relevant information of the patient. Thus, the use of the Center Method is considered, this method consists in the use of a set of validated forms where information relevant to the family, life history, and lifestyle of the patient with $\mathrm{AD}$ is gathered [12]. The forms would be filled semiautomatically in the system by the caregiver according to

\footnotetext{
${ }^{1}$ https://wiki.dbpedia.org/develop/datasets
} 
the information of the patient. Then the dialog model can use this information to generate personalized conversations according to such information.

\subsection{Conversational system}

As it was above-mentioned, the dialog model will be integrated into a conversational system prototype. The conversational system uses different modules to perform voice interaction to the user. Figure 2 describes the architecture of the system. First of all, the system must have an input mechanism for communication with the user, in this case, the Google Cloud Speech-to-Text $\mathrm{API}^{2}$ will be used within the Automatic Speech Recognition (ASR) module to transcript the utterances from the user to text. Subsequently, it is necessary to analyze the text and obtain a semantic interpretation of the information contained in the received message as it was already mentioned. All this process will be done in the Natural Language Understanding (NLU) module using a diverse type of techniques to perform this task. Some of this techniques imply named entity recognition, speech acts classification, etc.

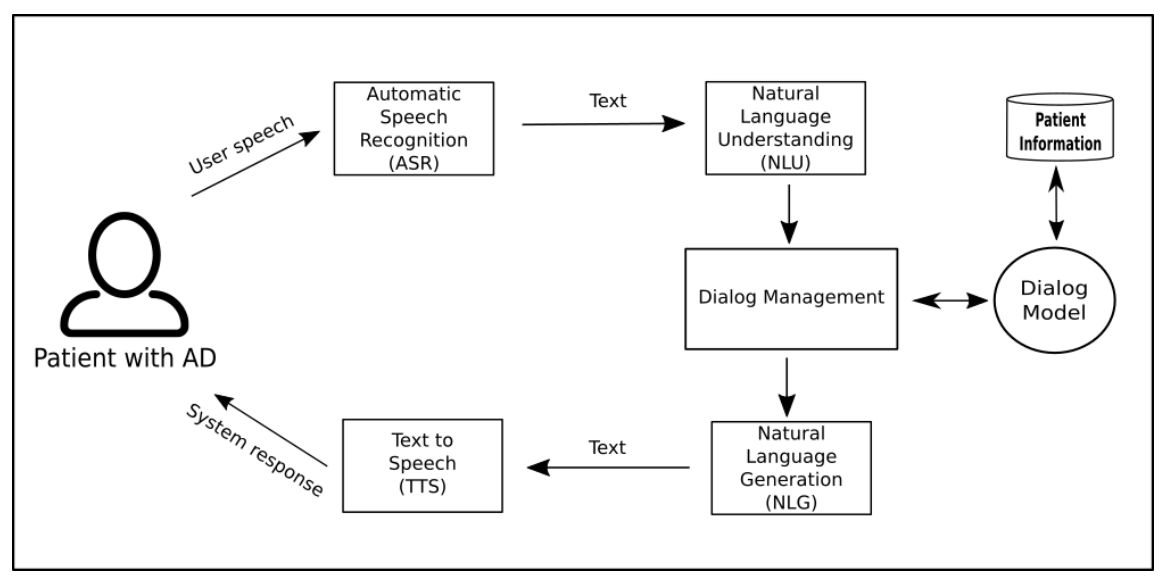

Fig. 2. Architecture of the conversational system proposed.

The Dialog Management module will be in charge of managing the turns of conversation between the user and the system and determining the actions to perform during the interaction according to the current state of the dialog. This module will interact directly with the Dialog Model to interchange semantic information that can be used to select the patient's information relevant for the conversation.

It is necessary to generate a response according to the information provided by the Dialog Model. The response by the system has to be understood by

\footnotetext{
${ }^{2}$ https://cloud.google.com/speech-to-text/
} 
the user. Hence, to represent in natural language the response provided by the Dialog Management module, diverse techniques will be implemented into the NLG module. Finally, the response will be synthesized through the TTS module using the IBM Watson Text-to-Speech $\mathrm{API}^{3}$ that offers a more natural-sounding audio.

With the objective to clarify how the system is expected to conduct the dialog with people with $\mathrm{AD}$, the following example in Figure 3 is given. The figure describes a particular conversation that could be performed by the system. First, the system asks the user: "¿Dónde naciste María? (Where were you born Maria?)". Suppose that the user María answers "Yo naci en la ciudad de Puebla (I was born in Puebla city)".

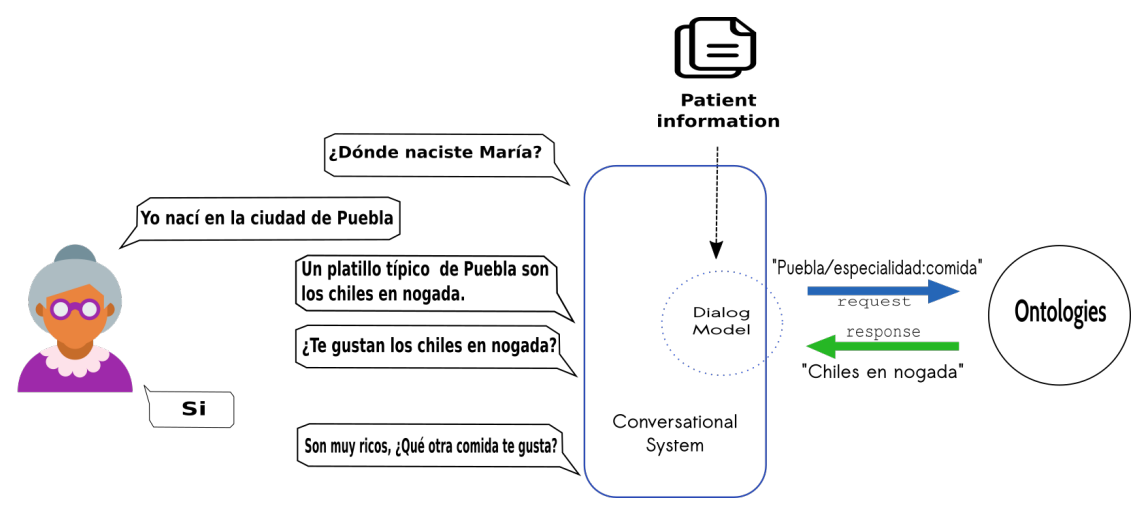

Fig. 3. Example of a dialog generated by the conversational system.

Then, the dialog model would apply some of the semantic rules established to extract the named entity of Puebla and to know that Puebla could be a city or a state. Based on that information a query over different ontologies will be performed to extract relevant information about Puebla. In this case, the query retrieved the traditional food of Puebla where a possible result would be chiles en nogada. Using this information, the model enriches the conversation by mentioning "Un platillo típico de Puebla son los chiles en nogada (A typical dish of Puebla are chiles en nogada)", and the system would extend the conversation by asking "¿Te gustan los chiles en nogada? (Do you like chiles en nogada?)", where the user could affirm "Si (Yes)" and the system would motivate to continue the conversation by saying "Son muy ricos. ¿Qué otra comida te gusta? (It is delicious. What other kind food do you like too?)".

\footnotetext{
$\overline{3}$ https://www.ibm.com/watson/services/text-to-speech/
} 


\section{Conclusion}

Due to the cognitive impairment that affects people with dementia, in early stage patients present different types of symptoms, one of the most common is short-term memory loss. This situation significantly affects their social behavior towards the people around them and generates unstable psychological states such as anxiety and depression. However, it has been observed that providing patients with non-pharmacological treatments such as reminiscence therapy improve psychological well-being. There are many proposals with the aim of supporting different kinds of therapy to address the psychological, emotional and behavioral symptoms of dementia. Most of them are based on an interaction with the patient through the use of generic dialogs used for all patients, thus the personalization in the intervention is lost.

In this paper, we propose the creation of a dialog model that could be integrated into a conversational system that generates individually personalized dialogs for each patient with AD according to their life history and preferences. Similarly, the model will enrich the dialogs through the use of ontologies that allow the extraction of relevant content associated with the information collected from the user.

In future work, it is initially contemplated to implement diverse natural language understanding techniques that allow us to realize a semantic analysis of the patient's utterances. As well, the selection of useful resources for dynamic dialog generation in real time by the system. Finally, it is considered to perform an experimental evaluation to asses the effectiveness and adoption of the system by people in the early stage of the AD.

\section{References}

1. Casey, D., Felzmann, H., Pegman, G., Kouroupetroglou, C., Murphy, K., Koumpis, A., Whelan, S.: What people with dementia want: designing mario an acceptable robot companion. In: International Conference on Computers Helping People with Special Needs. pp. 318-325. Springer (2016)

2. Chinaei, H., Currie, L.C., Danks, A., Lin, H., Mehta, T., Rudzicz, F.: Identifying and avoiding confusion in dialogue with people with alzheimer's disease. Computational Linguistics 43(2), 377-406 (2017)

3. Cruz-Sandoval, D., Favela, J.: Semi-autonomous conversational robot to deal with problematic behaviors from people with dementia. In: Ochoa, S.F., Singh, P., Bravo, J. (eds.) Ubiquitous Computing and Ambient Intelligence. pp. 677-688. Springer International Publishing, Cham (2017)

4. Fernández, P.H., Velarde, S.I., Hernández, M.F., Murguía, V.: Dinámica demográfica 1990-2010 y proyecciones de población 2010-2030. Consejo Nacional de Población (CONAPO) (2014), http://www . conapo.gob.mx/work/models/CONAPO/ Proyecciones/Cuadernos/15_Cuadernillo_Mexico.pdf

5. Gitlin, L.N., Hodgson, N., Jutkowitz, E., Pizzi, L.: The Cost-Effectiveness of a Nonpharmacologic Intervention for Individuals With Dementia and Family Caregivers: The Tailored Activity Program. The American Journal of Geriatric Psychiatry 18(6), 510-519 (jun 2010) 
6. González-Arévalo, K.A.: Terapia de reminiscencia y sus efectos en los pacientes mayores con demencia. Psicogeriatría 5, 101-11 (2015)

7. Gutierrez, L.: Demencias en México. In: Demencias, una visión panorámica, pp. 9-20. Universitaria Potosina (2014)

8. Navarro, R.F., Rodríguez, M.D., Favela, J.: Use and adoption of an assisted cognition system to support therapies for people with dementia. Computational and Mathematical Methods in Medicine 2016 (2016)

9. Neal, M., Barton Wright, P.: Validation therapy for dementia. The Cochrane Library (2003), art. No.: CD001394. DOI: 10.1002/14651858.CD001394

10. Perilli, V., Lancioni, G.E., Hoogeveen, F., Caffó, A., Singh, N., O'Reilly, M., Sigafoos, J., Cassano, G., Oliva, D.: Video prompting versus other instruction strategies for persons with alzheimer's disease. American Journal of Alzheimer's Disease and other Dementias 28(4), 393-402 (jun 2013), http://journals. sagepub.com/doi/10.1177/1533317513488913

11. Rodriguez, J.J.L., Ferri, C.P., Acosta, D., Guerra, M., Huang, Y., Jacob, K., Krishnamoorthy, E., Salas, A., Sosa, A.L., Acosta, I., et al.: Prevalence of dementia in latin america, india, and china: a population-based cross-sectional survey. The Lancet 372(9637), 464-474 (2008)

12. Rokkaku, R.: Care management sheet pack for the elderly with dementia: The center method ver. 03. Nihon Ronen Igakkai zasshi. Japanese journal of geriatrics 42(3), 318-319 (2005)

13. Rudzicz, F., Wang, R., Begum, M., Mihailidis, A.: Speech Interaction with Personal Assistive Robots Supporting Aging at Home for Individuals with Alzheimer's Disease. ACM Transactions on Accessible Computing 7(2), 1-22 (may 2015), http://dl . acm.org/citation. cfm?doid=2785580.2744206

14. Sakakibara, S., Saiki, S., Nakamura, M., Yasuda, K.: Generating personalized dialogue towards daily counseling system for home dementia care. In: Duffy, V.G. (ed.) Digital Human Modeling. Applications in Health, Safety, Ergonomics, and Risk Management: Health and Safety. pp. 161-172. Springer International Publishing, Cham (2017)

15. Sarabia, M., Young, N., Canavan, K., Edginton, T., Demiris, Y., Vizcaychipi, M.P.: Assistive Robotic Technology to Combat Social Isolation in Acute Hospital Settings. International Journal of Social Robotics (2018), http://link.springer. com/10.1007/s12369-017-0421-z

16. Tanaka, H., Adachi, H., Ukita, N., Ikeda, M., Kazui, H., Kudo, T., Nakamura, S.: Detecting dementia through interactive computer avatars. IEEE journal of translational engineering in health and medicine 5, 1-11 (2017)

17. Wimo, A., Prince, M.: World Alzheimer Report 2010. Tech. rep., Alzheimer's Disease International (2010)

18. Wolters, M.K., Kelly, F., Kilgour, J.: Designing a spoken dialogue interface to an intelligent cognitive assistant for people with dementia. Health Informatics Journal $22(4), 854-866(2016)$

19. Woods, B., Spector, A.E., Jones, C.A., Orrell, M., Davies, S.P.: Reminiscence therapy for dementia. The Cochrane Library (2005)

20. Yasuda, K., Kuwahara, N., Kuwabara, K., Morimoto, K., Tetsutani, N.: Daily assistance for individuals with dementia via videophone. American Journal of Alzheimer's Disease and other Dementias 28(5), 508-516 (aug 2013) 